$\mathrm{J}$ o u r n a l of

Mathematics

and Applications

JMA No 39, pp 5-22 (2016)

\title{
Some New Existence Results and Stability Concepts for Fractional Partial Random Differential Equations
}

\author{
Saïd Abbas, Mouffak Benchohra and Mohamed Abdalla \\ Darwish
}

\begin{abstract}
In the present paper we provide some existence results and Ulam's type stability concepts for the Darboux problem of partial fractional random differential equations in Banach spaces, by applying the measure of noncompactness and a random fixed point theorem with stochastic domain.
\end{abstract}

AMS Subject Classification: 26A33, 34G20, 34A40, 45N05, 47H10.

Keywords and Phrases: Random differential equation; Left-sided mixed RiemannLiouville integral; Caputo fractional order derivative; Banach space; Darboux problem; Ulam stability.

\section{Introduction}

The fractional calculus deals with extensions of derivatives and integrals to noninteger orders. The field of fractional differential equations has been subjected to an intensive development of the theory and the applications in mathematical physics, finance, hydrology, biophysics, thermodynamics, control theory, statistical mechanics, astrophysics, cosmology and bioengineering [22, 36, 42]. There has been a significant development in ordinary and partial fractional differential equations in recent years; see the monographs of Abbas et al. [4, 5], Kilbas et al. [29], and Zhou [51], the papers of Abbas et al. [1, 2, 3, 6, 7, 8], Baleanu et al. [11], Darwish et al. [16, 17, 18], Vityuk et al. $[44,45,46]$, and the references therein.

On the other hand, due to a combination of uncertainties and complexities, deterministic equations can hardly describe a real system precisely. In order to take random factors into account, many stochastic models were proposed and various achievements were obtained; see for instance the book by Soon [41], and the references therein.

COPYRIGHT (c) by Publishing House of Rzeszów University of Technology P.O. Box 85, 35-959 Rzeszów, Poland 
The initial value problems of ordinary random differential equations have been studied in the literature on bounded as well as unbounded intervals of the real line for different aspects of the solution. See for example, Burton and Furumochi [14] and the references therein.

The stability of functional equations was originally raised by Ulam in 1940 in a talk given at Wisconsin University. The problem posed by Ulam was the following: Under what conditions does there exist an additive mapping near an approximately additive mapping? (for more details see [43]). The first answer to Ulam's question was given by Hyers in 1941 in the case of Banach spaces [23]. Thereafter, this type of stability is called the Ulam-Hyers stability. In 1978, Rassias [38] provided a remarkable generalization of the Ulam-Hyers stability of mappings by considering variables. The concept of stability for a functional equation arises when we replace the functional equation by an inequality which acts as a perturbation of the equation. Thus, the stability question of functional equations is how do the solutions of the inequality differ from those of the given functional equation? Considerable attention has been given to the study of the Ulam-Hyers and Ulam-Hyers-Rassias stability of all kinds of functional equations; see the monographs [24, 26]. Bota-Boriceanu and Petrusel [13], Petru et al. [34, 35], and Rus [39, 40] discussed the Ulam-Hyers stability for operatorial equations and inclusions. Castro and Ramos [15], and Jung [28] considered the HyersUlam-Rassias stability for a class of Volterra integral equations. Ulam stability for fractional differential equations with Caputo derivative are proposed by Wang et al. $[47,48]$. Some stability results for fractional integral equation are obtained by Wei et al. [49]. More details from historical point of view, and recent developments of such stabilities are reported in $[27,39,49]$.

In this paper, we discuss the existence of random solutions and Ulam stabilities for the following fractional partial random differential equations

$$
{ }^{c} D_{\theta}^{r} u(x, y, w)=f(x, y, u(x, y, w), w) ; \text { for a.a. }(x, y) \in J:=[0, a] \times[0, b], w \in \Omega,
$$

with the initial conditions

$$
\left\{\begin{array}{l}
u(x, 0, w)=\varphi(x, w) ; x \in[0, a] \\
u(0, y, w)=\psi(y, w) ; y \in[0, b], \quad w \in \Omega \\
\varphi(0, w)=\psi(0, w)
\end{array}\right.
$$

where $a, b>0, \theta=(0,0),{ }^{c} D_{\theta}^{r}$ is the fractional Caputo derivative of order $r=$ $\left(r_{1}, r_{2}\right) \in(0,1] \times(0,1],(\Omega, \mathcal{A})$ is a measurable space, $f: J \times E \times \Omega \rightarrow E$ is a given continuous function, $\left(E,\|\cdot\|_{E}\right)$ is a real Banach space, $\varphi:[0, a] \times \Omega \rightarrow E$, $\psi:[0, b] \times \Omega \rightarrow E$ are given functions such that $\varphi(\cdot, w)$ and $\psi(\cdot, w)$ are absolutely continuous functions for all $w \in \Omega$, and $\varphi(x, \cdot)$ and $\psi(y, \cdot)$ are measurable for all $x \in[0, a]$ and $y \in[0, b]$ respectively, and $\mathcal{C}$ is the Banach space of all continuous functions from $J$ into $E$ with the supremum (uniform) norm $\|\cdot\|_{\infty}$. This paper initiates the existence and Ulam stabilities of random solutions via fixed point techniques. 


\section{Preliminaries}

In this section, we introduce notations, definitions, and preliminary facts which are used throughout this paper. Denote $L^{1}(J)$ the space of Bochner-integrable functions $u: J \rightarrow E$ with the norm

$$
\|u\|_{L^{1}}=\int_{0}^{a} \int_{0}^{b}\|u(x, y)\|_{E} d y d x .
$$

$L^{\infty}(J)$ the Banach space of functions $u: J \rightarrow \mathbb{R}$ which are essentially bounded.

As usual, by $A C(J)$ we denote the space of absolutely continuous functions from $J$ into $E$.

Let $\beta_{E}$ be the $\sigma$-algebra of Borel subsets of $E$. A mapping $v: \Omega \rightarrow E$ is said to be measurable if for any $B \in \beta_{E}$, one has

$$
v^{-1}(B)=\{w \in \Omega: v(w) \in B\} \subset \mathcal{A} .
$$

To define integrals of sample paths of random process, it is necessary to define a jointly measurable map.

Definition 2.1. A mapping $T: \Omega \times E \rightarrow E$ is called jointly measurable if for any $B \in \beta_{E}$, one has

$$
T^{-1}(B)=\{(w, v) \in \Omega \times E: T(w, v) \in B\} \subset \mathcal{A} \times \beta_{E},
$$

where $\mathcal{A} \times \beta_{E}$ is the direct product of the $\sigma$-algebras $\mathcal{A}$ and $\beta_{E}$ those defined in $\Omega$ and $E$ respectively.

Lemma 2.2. [19] Let $T: \Omega \times E \rightarrow E$ be a mapping such that $T(\cdot, v)$ is measurable for all $v \in E$, and $T(w, \cdot)$ is continuous for all $w \in \Omega$. Then the map $(w, v) \mapsto T(w, v)$ is jointly measurable.

Definition 2.3. [21] A function $f: J \times E \times \Omega \rightarrow E$ is called random Carathéodory if the following conditions are satisfied:

(i) The map $(x, y, w) \rightarrow f(x, y, u, w)$ is jointly measurable for all $u \in E$, and

(ii) The map $u \rightarrow f(x, y, u, w)$ is continuous for almost all $(x, y) \in J$ and $w \in \Omega$.

Let $T: \Omega \times E \rightarrow E$ be a mapping. Then $T$ is called a random operator if $T(w, u)$ is measurable in $w$ for all $u \in E$ and it is expressed as $T(w) u=T(w, u)$. In this case we also say that $T(w)$ is a random operator on $E$. A random operator $T(w)$ on $E$ is called continuous (resp. compact, totally bounded and completely continuous) if $T(w, u)$ is continuous (resp. compact, totally bounded and completely continuous) in $u$ for all $w \in \Omega$. The details of completely continuous random operators in Banach spaces and their properties appear in Itoh [25]. 
Definition 2.4. [20] Let $\mathcal{P}(Y)$ be the family of all nonempty subsets of $Y$ and $C$ be a mapping from $\Omega$ into $\mathcal{P}(Y)$. A mapping $T:\{(w, y): w \in \Omega, y \in C(w)\} \rightarrow Y$ is called random operator with stochastic domain $C$ if $C$ is measurable (i.e., for all closed $A \subset Y,\{w \in \Omega, C(w) \cap A \neq \emptyset\}$ is measurable) and for all open $D \subset Y$ and all $y \in Y,\{w \in \Omega: y \in C(w), T(w, y) \in D\}$ is measurable. $T$ will be called continuous if every $T(w)$ is continuous. For a random operator $T$, a mapping $y: \Omega \rightarrow Y$ is called random (stochastic) fixed point of $T$ if for $P$-almost all $w \in \Omega, y(w) \in C(w)$ and $T(w) y(w)=y(w)$ and for all open $D \subset Y,\{w \in \Omega: y(w) \in D\}$ is measurable.

Let $\mathcal{M}_{X}$ denote the class of all bounded subsets of a metric space $X$.

Definition 2.5. Let $X$ be a complete metric space. A map $\alpha: \mathcal{M}_{X} \rightarrow[0, \infty)$ is called a measure of noncompactness on $X$ if it satisfies the following properties for all $B, B_{1}, B_{2} \in \mathcal{M}_{X}$.

(MNC.1) $\alpha(B)=0$ if and only if $B$ is precompact (Regularity),

(MNC.2) $\alpha(B)=\alpha(\bar{B})$ (Invariance under closure),

(MNC.3) $\alpha\left(B_{1} \cup B_{2}\right)=\alpha\left(B_{1}\right)+\alpha\left(B_{2}\right)$ (Semi-additivity).

For more details on measure of noncompactness and its properties see [9].

Example 2.6. In every metric space $X$, the map $\phi: \mathcal{M}_{X} \rightarrow[0, \infty)$ with $\phi(B)=0$ if $B$ is relatively compact and $\phi(B)=1$ otherwise is a measure of noncompactness, the so-called discrete measure of noncompactness [[10], Example1, p. 19].

Let $\theta=(0,0), r_{1}, r_{2}>0$ and $r=\left(r_{1}, r_{2}\right)$. For $f \in L^{1}(J)$, the expression

$$
\left(I_{\theta}^{r} f\right)(x, y)=\frac{1}{\Gamma\left(r_{1}\right) \Gamma\left(r_{2}\right)} \int_{0}^{x} \int_{0}^{y}(x-s)^{r_{1}-1}(y-t)^{r_{2}-1} f(s, t) d t d s
$$

is called the left-sided mixed Riemann-Liouville integral of order $r$, where $\Gamma(\cdot)$ is the (Euler's) Gamma function defined by $\Gamma(\xi)=\int_{0}^{\infty} t^{\xi-1} e^{-t} d t ; \xi>0$.

In particular,

$$
\left(I_{\theta}^{\theta} u\right)(x, y)=u(x, y),\left(I_{\theta}^{\sigma} u\right)(x, y)=\int_{0}^{x} \int_{0}^{y} u(s, t) d t d s ; \text { for almost all }(x, y) \in J
$$

where $\sigma=(1,1)$.

For instance, $I_{\theta}^{r} u$ exists for all $r_{1}, r_{2} \in(0, \infty)$, when $u \in L^{1}(J)$. Note also that when $u \in \mathcal{C}$, then $\left(I_{\theta}^{r} u\right) \in \mathcal{C}$, moreover

$$
\left(I_{\theta}^{r} u\right)(x, 0)=\left(I_{\theta}^{r} u\right)(0, y)=0 ; x \in[0, a], y \in[0, b] .
$$

By $1-r$ we mean $\left(1-r_{1}, 1-r_{2}\right) \in[0,1) \times[0,1)$. Denote by $D_{x y}^{2}:=\frac{\partial^{2}}{\partial x \partial y}$, the mixed second order partial derivative. 
Definition 2.7. [4, 46] Let $r \in(0,1] \times(0,1]$ and $u \in L^{1}(J)$. The Caputo fractionalorder derivative of order $r$ of $u$ is defined by the expression

$$
{ }^{c} D_{\theta}^{r} u(x, y)=\left(I_{\theta}^{1-r} D_{x y}^{2} u\right)(x, y) .
$$

The case $\sigma=(1,1)$ is included and we have

$$
\left({ }^{c} D_{\theta}^{\sigma} u\right)(x, y)=\left(D_{x y}^{2} u\right)(x, y) ; \text { for almost all }(x, y) \in J .
$$

Definition 2.8. By a random solution of the random problem (1)-(2) we mean a measurable function $u: \Omega \rightarrow A C(J)$ that satisfies the equation (1) a.a. on $J \times \Omega$ and the initial conditions (2) are satisfied.

Let $h \in L^{1}\left(J, \mathbb{R}^{n}\right)$. We need the following lemma:

Lemma 2.9. [1, 4] A function $u \in A C\left(J, \mathbb{R}^{n}\right)$ is a solution of problem

$$
\left\{\begin{array}{l}
{ }^{c} D_{\theta}^{r} u(x, y)=h(x, y) ; \text { for a.a. }(x, y) \in J:=[0, a] \times[0, b] \\
u(x, 0)=\varphi(x) ; x \in[0, a] \\
u(0, y)=\psi(y) ; y \in[0, b] \\
\varphi(0)=\psi(0) .
\end{array}\right.
$$

if and only if $u$ satisfies

$$
u(x, y)=\mu(x, y)+I_{\theta}^{r} h(x, y) ; \text { for a.a. }(x, y) \in J,
$$

where

$$
\mu(x, y)=\varphi(x)+\psi(y)-\varphi(0)
$$

Let us assume that the function $f$ is random Carathéodory on $J \times E \times \Omega$. From the above lemma, we have the following Lemma.

Lemma 2.10. Let $0<r_{1}, r_{2} \leq 1$. A function $u \in \Omega \times A C$ is a solution of the random fractional integral equation

$u(x, y, w)=\mu(x, y, w)+\frac{1}{\Gamma\left(r_{1}\right) \Gamma\left(r_{2}\right)} \int_{0}^{x} \int_{0}^{y}(x-s)^{r_{1}-1}(y-t)^{r_{2}-1} f(s, t, u(s, t, w), w) d t d s$,

where

$$
\mu(x, y, w)=\varphi(x, w)+\psi(y, w)-\varphi(0, w),
$$

if and only if $u$ is a solution of the random problem (1)-(2).

Now, we consider the Ulam stability of fractional random differential equation (1). Let $\epsilon$ be a positive real number and $\Phi: J \times \Omega \rightarrow[0, \infty)$ be a measurable and bounded function. We consider the following inequalities

$$
\begin{gathered}
\left\|{ }^{c} D_{\theta}^{r} u(x, y, w)-f(x, y, u(x, y, w), w)\right\|_{E} \leq \epsilon ; \text { for a.a. }(x, y) \in J, w \in \Omega . \\
\left\|{ }^{c} D_{\theta}^{r} u(x, y, w)-f(x, y, u(x, y, w), w)\right\|_{E} \leq \Phi(x, y, w) ; \text { for a.a. }(x, y) \in J, w \in \Omega . \\
\left\|{ }^{c} D_{\theta}^{r} u(x, y, w)-f(x, y, u(x, y, w), w)\right\|_{E} \leq \epsilon \Phi(x, y, w) ; \text { for a.a. }(x, y) \in J, w \in \Omega .
\end{gathered}
$$


Definition 2.11. The random equation (1) is Ulam-Hyers stable if there exists a real number $c_{f}>0$ such that for each $\epsilon>0$ and for each random solution $u: \Omega \rightarrow A C(J)$ of the inequality (4), there exists a random solution $v: \Omega \rightarrow A C(J)$ of problem (1) with

$$
\|u(x, y, w)-v(x, y, w)\|_{E} \leq \epsilon c_{f} ; \quad(x, y) \in J, w \in \Omega .
$$

Definition 2.12. The random equation (1) is generalized Ulam-Hyers stable if there exists $\theta_{f} \in C([0, \infty),[0, \infty)), \theta_{f}(0)=0$ such that for each $\epsilon>0$ and for each random solution $u: \Omega \rightarrow A C(J)$ of the inequality (4), there exists a random solution $v: \Omega \rightarrow A C(J)$ of problem (1) with

$$
\|u(x, y, w)-v(x, y, w)\|_{E} \leq \theta_{f}(\epsilon) ;(x, y) \in J, w \in \Omega .
$$

Definition 2.13. The random equation (1) is Ulam-Hyers-Rassias stable with respect to $\Phi$ if there exists a real number $c_{f, \Phi}>0$ such that for each $\epsilon>0$ and for each random solution $u: \Omega \rightarrow A C(J)$ of the inequality (6), there exists a random solution $v: \Omega \rightarrow A C(J)$ of problem (1) with

$$
\|u(x, y, w)-v(x, y, w)\|_{E} \leq \epsilon c_{f, \Phi} \Phi(x, y, w) ;(x, y) \in J, w \in \Omega .
$$

Definition 2.14. The random equation (1) is generalized Ulam-Hyers-Rassias stable with respect to $\Phi$ if there exists a real number $c_{f, \Phi}>0$ such that for each random solution $u: \Omega \rightarrow A C(J)$ of the inequality (5), there exists a random solution $v: \Omega \rightarrow A C(J)$ of problem (1) with

$$
\|u(x, y, w)-v(x, y, w)\|_{E} \leq c_{f, \Phi} \Phi(x, y, w) ;(x, y) \in J, w \in \Omega .
$$

Remark 2.15. It is clear that

(i) Definition $2.11 \Rightarrow$ Definition 2.12,

(ii) Definition $2.13 \Rightarrow$ Definition 2.14,

(iii) Definition 2.13 for $\Phi(x, y)=1 \Rightarrow$ Definition 2.11 .

Remark 2.16. A function $u: \Omega \rightarrow A C(J)$ is a solution of the inequality (4) if and only if there exists a function $g: \Omega \rightarrow C(J)$ (which depends on u) such that

(i) $\|g(x, y, w)\|_{E} \leq \epsilon$,

(ii) ${ }^{c} D_{\theta}^{r} u(x, y, w)=f(x, y, u(x, y, w), w)+g(x, y, w) ; \quad$ a.a. $(x, y) \in J, w \in \Omega$.

One can have similar remarks for the inequalities (5) and (6). So, the Ulam stabilities of the fractional random differential equations are some special types of data dependence of the solutions of fractional differential equations.

Lemma 2.17. [12] If $Y$ is a bounded subset of Banach space $X$, then for each $\epsilon>0$, there is a sequence $\left\{y_{k}\right\}_{k=1}^{\infty} \subset Y$ such that

$$
\alpha(Y) \leq 2 \alpha\left(\left\{y_{k}\right\}_{k=1}^{\infty}\right)+\epsilon .
$$


Lemma 2.18. [31, 50] If $\left\{u_{k}\right\}_{k=1}^{\infty} \subset L^{1}(J)$ is uniformly integrable, then $\alpha\left(\left\{u_{k}\right\}_{k=1}^{\infty}\right)$ is measurable and for each $(x, y) \in J$,

$$
\alpha\left(\left\{\int_{0}^{x} \int_{0}^{y} u_{k}(s, t) d t d s\right\}_{k=1}^{\infty}\right) \leq 2 \int_{0}^{x} \int_{0}^{y} \alpha\left(\left\{u_{k}(s, t)\right\}_{k=1}^{\infty}\right) d t d s .
$$

Lemma 2.19. [30] Let $F$ be a closed and convex subset of a real Banach space, let $G: F \rightarrow F$ be a continuous operator and $G(F)$ be bounded. If there exist a constant $k \in[0,1)$ such that for each bounded subset $B \subset F$,

$$
\alpha(G(B)) \leq k \alpha(B)
$$

then $G$ has a fixed point in $F$.

In the sequel we will make use of the following generalization of Gronwall's lemma.

Lemma 2.20. (Gronwall lemma) [32, 33] Let $v: J \times \Omega \rightarrow[0, \infty)$ be a real function and $\omega(x, y, w)$ be a measurable, nonnegative and locally integrable function on $J \times \Omega$. If there are constants $c>0$ and $0<r_{1}, r_{2}<1$ such that

$$
v(x, y, w) \leq \omega(x, y, w)+c \int_{0}^{x} \int_{0}^{y} \frac{v(s, t, w)}{(x-s)^{r_{1}}(y-t)^{r_{2}}} d t d s
$$

then there exists a constant $\delta=\delta\left(r_{1}, r_{2}\right)$ such that

$$
v(x, y, w) \leq \omega(x, y, w)+\delta c \int_{0}^{x} \int_{0}^{y} \frac{\omega(s, t, w)}{(x-s)^{r_{1}}(y-t)^{r_{2}}} d t d s,
$$

for every $(x, y) \in J$ and $w \in \Omega$.

\section{Existence and stability Results}

In this section, we discuss the existence of random solutions and we present conditions for the Ulam stability for the problem (1)-(2).

Lemma 3.1. If $u: \Omega \rightarrow A C(J)$ is a solution of the inequality (4) then $u$ is a solution of the following integral inequality

$$
\begin{gathered}
\left\|u(x, y, w)-\mu(x, y, w)-\int_{0}^{x} \int_{0}^{y} \frac{(x-s)^{r_{1}-1}(y-t)^{r_{2}-1}}{\Gamma\left(r_{1}\right) \Gamma\left(r_{2}\right)} f(s, t, u(s, t, w), w) d t d s\right\|_{E} \\
\leq \frac{\epsilon a^{r_{1}} b^{r_{2}}}{\Gamma\left(1+r_{1}\right) \Gamma\left(1+r_{2}\right)} ; \text { if }(x, y) \in J, w \in \Omega
\end{gathered}
$$

Proof. By Remark 2.16, for $(x, y) \in J$ and $w \in \Omega$ there exists $g: \Omega \rightarrow C(J)$ such that

$$
{ }^{c} D_{\theta}^{r} u(x, y, w)=f(x, y, u(x, y, w), w)+g(x, y, w) .
$$


Then, for each $(x, y) \in J$ and $w \in \Omega$, we get

$$
\begin{gathered}
u(x, y, w)=\mu(x, y, w) \\
+\int_{0}^{x} \int_{0}^{y} \frac{(x-s)^{r_{1}-1}(y-t)^{r_{2}-1}}{\Gamma\left(r_{1}\right) \Gamma\left(r_{2}\right)}[g(s, t, w)+f(s, t, u(s, t, w), w)] d t d s .
\end{gathered}
$$

Thus, for each $(x, y) \in J$ and $w \in \Omega$, we obtain

$$
\begin{aligned}
& \left\|u(x, y, w)-\mu(x, y, w)-\int_{0}^{x} \int_{0}^{y} \frac{(x-s)^{r_{1}-1}(y-t)^{r_{2}-1}}{\Gamma\left(r_{1}\right) \Gamma\left(r_{2}\right)} f(s, t, u(s, t, w), w) d t d s\right\|_{E} \\
& =\left\|\frac{1}{\Gamma\left(r_{1}\right) \Gamma\left(r_{2}\right)} \int_{0}^{x} \int_{0}^{y}(x-s)^{r_{1}-1}(y-t)^{r_{2}-1} g(s, t, w) d t d s\right\|_{E} \\
& \leq \frac{\epsilon a^{r_{1}} b^{r_{2}}}{\Gamma\left(1+r_{1}\right) \Gamma\left(1+r_{2}\right)} .
\end{aligned}
$$

Hence, we obtain (7).

Remark 3.2. One can obtain similar results for the solutions of the inequalities (5) and (6).

The following hypotheses will be used in the sequel.

$\left(H_{1}\right)$ The functions $w \mapsto \varphi(x, 0, w)$ and $w \mapsto \psi(0, y, w)$ are measurable and bounded for a.e. $x \in[0, a]$ and $y \in[0, b]$ respectively,

$\left(H_{2}\right)$ The function $f$ is random Carathéodory on $J \times E \times \Omega$,

$\left(H_{3}\right)$ There exist functions $p_{1}, p_{2}: J \times \Omega \rightarrow[0, \infty)$ with $p_{i}(\cdot, w) \in L^{\infty}(J,[0, \infty))$; $i=1,2$ such that for each $w \in \Omega$,

$$
\|f(x, y, u, w)\|_{E} \leq p_{1}(x, y, w)+p_{2}(x, y, w)\|u\|_{E},
$$

for all $u \in E$ and a.e. $(x, y) \in J$,

$\left(H_{4}\right)$ There exists a function $q: J \times \Omega \rightarrow[0, \infty)$ with $q(\cdot, w) \in L^{\infty}(J,[0, \infty))$ for each $w \in \Omega$ such that for any bounded $B \subset E$,

$$
\alpha(f(x, y, B, w)) \leq q(x, y, w) \alpha(B), \text { for a.e. }(x, y) \in J
$$

$\left(H_{5}\right)$ There exists a random function $R: \Omega \rightarrow(0, \infty)$ such that

$$
\mu^{*}(w)+\frac{\left(p_{1}^{*}(w)+p_{2}^{*}(w) R(w)\right) a^{r_{1}} b^{r_{2}}}{\Gamma\left(1+r_{1}\right) \Gamma\left(1+r_{2}\right)} \leq R(w)
$$

where

$$
\mu^{*}(w)=\sup _{(x, y) \in J}\|\mu(x, y, w)\|_{E}, p_{i}^{*}(w)=\sup _{(x, y) \in J} p_{i}(x, y, w) ; i=1,2,
$$


$\left(H_{6}\right)$ The function $f$ satisfies

$$
\|f(x, y, u, w)-f(x, y, \bar{u}, w)\|_{E} \leq q(x, y, w)\|u-\bar{u}\|_{E},
$$

for each $(x, y) \in J, w \in \Omega$ and $u, \bar{u} \in E$,

$\left(H_{7}\right) \Phi(w) \in L^{1}(J,[0, \infty))$ for all $w \in \Omega$, and there exists $\lambda_{\Phi}>0$ such that, for each $(x, y) \in J$ we have

$$
\left(I_{\theta}^{r} \Phi\right)(x, y, w) \leq \lambda_{\Phi} \Phi(x, y, w)
$$

\section{Remark 3.3.}

1. Hypothesis $\left(H_{6}\right)$ implies hypothesis $\left(H_{3}\right)$, with

$$
p_{1}(x, y, w)=\|f(x, y, 0, w)\|, \text { and } p_{2}(x, y, w)=q(x, y, w) .
$$

2. Hypotheses $\left(H_{4}\right)$ and $\left(H_{6}\right)$ are equivalent $([9])$.

Set

$$
q^{*}=\sup _{(x, y, w) \in J \times \Omega} \operatorname{ess}(x, y, w) .
$$

Theorem 3.4. Assume that hypotheses $\left(H_{1}\right)-\left(H_{5}\right)$ hold. If

$$
\ell:=\frac{4 q^{*} a^{r_{1}} b^{r_{2}}}{\Gamma\left(1+r_{1}\right) \Gamma\left(1+r_{2}\right)}<1
$$

then the problem (1)-(2) has a random solution defined on J.

Proof. From hypotheses $\left(H_{2}\right),\left(H_{3}\right)$, for each $w \in \Omega$ and almost all $(x, y) \in J$, we have that $f(x, y, u(x, y, w), w)$ is in $L^{1}$. By using Lemma 2.10, the problem (1)-(2) is equivalent to the integral equation

$$
u(x, y, w)=\mu(x, y, w)+\int_{0}^{x} \int_{0}^{y} \frac{(x-s)^{r_{1}-1}(y-t)^{r_{2}-1}}{\Gamma\left(r_{1}\right) \Gamma\left(r_{2}\right)} f(s, t, u(s, t, w), w) d t d s ;
$$

for each $w \in \Omega$ and a.e. $(x, y) \in J$.

Define the operator $N: \Omega \times \mathcal{C} \rightarrow \mathcal{C}$ by

$$
(N(w) u)(x, y)=\mu(x, y, w)+\int_{0}^{x} \int_{0}^{y} \frac{(x-s)^{r_{1}-1}(y-t)^{r_{2}-1}}{\Gamma\left(r_{1}\right) \Gamma\left(r_{2}\right)} f(s, t, u(s, t, w), w) d t d s .
$$

Since the functions $\varphi, \psi$ and $f$ are absolutely continuous, then the function $\mu$ and the indefinite integral are absolutely continuous for all $w \in \Omega$ and almost all $(x, y) \in J$. Again, as the map $\mu$ is continuous for all $w \in \Omega$ and the indefinite integral is continuous on $J$, then $N(w)$ defines a mapping $N: \Omega \times \mathcal{C} \rightarrow \mathcal{C}$. Hence $u$ is a solution for the problem (1)-(2) if and only if $u=(N(w)) u$. We shall show that the operator $N$ satisfies all conditions of Lemma 2.19. The proof will be given in several steps. 
Step 1: $N(w)$ is a random operator with stochastic domain on $\mathcal{C}$.

Since $f(x, y, u, w)$ is random Carathéodory, the map $w \rightarrow f(x, y, u, w)$ is measurable in view of Definition 2.1. Similarly, the product $(x-s)^{r_{1}-1}(y-t)^{r_{2}-1} f(s, t, u(s, t, w), w)$ of a continuous and a measurable function is again measurable. Further, the integral is a limit of a finite sum of measurable functions, therefore, the map

$$
w \mapsto \mu(x, y, w)+\frac{1}{\Gamma\left(r_{1}\right) \Gamma\left(r_{2}\right)} \int_{0}^{x} \int_{0}^{y}(x-s)^{r_{1}-1}(y-t)^{r_{2}-1} f(s, t, u(s, t, w), w) d t d s,
$$

is measurable. As a result, $N$ is a random operator on $\Omega \times \mathcal{C}$ into $\mathcal{C}$.

Let $W: \Omega \rightarrow \mathcal{P}(\mathcal{C})$ be defined by

$$
W(w)=\left\{u \in \mathcal{C}:\|u\|_{\infty} \leq R(w)\right\}
$$

with $W(w)$ bounded, closed, convex and solid for all $w \in \Omega$. Then $W$ is measurable by Lemma [[20], Lemma 17]. Let $w \in \Omega$ be fixed, then from $\left(H_{3}\right)$ and $\left(H_{5}\right)$ for any $u \in W(w)$, we get

$$
\begin{aligned}
\| & (N(w) u)(x, y) \|_{E} \\
\leq & \|\mu(x, y, w)\|_{E}+\int_{0}^{x} \int_{0}^{y} \frac{(x-s)^{r_{1}-1}(y-t)^{r_{2}-1}}{\Gamma\left(r_{1}\right) \Gamma\left(r_{2}\right)}\|f(s, t, u(s, t, w), w)\|_{E} d t d s \\
\leq & \|\mu(x, y, w)\|_{E}+\frac{1}{\Gamma\left(r_{1}\right) \Gamma\left(r_{2}\right)} \int_{0}^{x} \int_{0}^{y}(x-s)^{r_{1}-1}(y-t)^{r_{2}-1} p_{1}(s, t, w) d t d s \\
& +\frac{1}{\Gamma\left(r_{1}\right) \Gamma\left(r_{2}\right)} \int_{0}^{x} \int_{0}^{y}(x-s)^{r_{1}-1}(y-t)^{r_{2}-1} p_{2}(s, t, w)\|u(s, t, w)\|_{E} d t d s \\
\leq & \mu^{*}(w)+\frac{p_{1}^{*}(w)}{\Gamma\left(r_{1}\right) \Gamma\left(r_{2}\right)} \int_{0}^{x} \int_{0}^{y}(x-s)^{r_{1}-1}(y-t)^{r_{2}-1} d t d s \\
& +\frac{p_{2}^{*}(w) R(w)}{\Gamma\left(r_{1}\right) \Gamma\left(r_{2}\right)} \int_{0}^{x} \int_{0}^{y}(x-s)^{r_{1}-1}(y-t)^{r_{2}-1} d t d s \\
\leq & \mu^{*}(w)+\frac{\left(p_{1}^{*}(w)+p_{2}^{*}(w) R(w)\right) a^{r_{1}} b^{r_{2}}}{\Gamma\left(1+r_{1}\right) \Gamma\left(1+r_{2}\right)} \\
\leq & R(w) .
\end{aligned}
$$

Therefore, $N$ is a random operator with stochastic domain $W$ and $N(w): W(w) \rightarrow$ $W(w)$. Furthermore, $N(w)$ maps bounded sets into bounded sets in $\mathcal{C}$.

Step 2: $N(w)$ is continuous.

Let $\left\{u_{n}\right\}$ be a sequence such that $u_{n} \rightarrow u$ in $\mathcal{C}$. Then, for each $(x, y) \in J$ and $w \in \Omega$, we have

$$
\begin{aligned}
& \left\|\left(N(w) u_{n}\right)(x, y)-(N(w) u)(x, y)\right\|_{E} \leq \frac{1}{\Gamma\left(r_{1}\right) \Gamma\left(r_{2}\right)} \int_{0}^{x} \int_{0}^{y}(x-s)^{r_{1}-1}(y-t)^{r_{2}-1} \\
& \times\left\|f\left(s, t, u_{n}(s, t, w), w\right)-f(s, t, u(s, t, w), w)\right\|_{E} d t d s
\end{aligned}
$$

Using the Lebesgue Dominated Convergence Theorem, we get

$$
\left\|N(w) u_{n}-N(w) u\right\|_{\infty} \rightarrow 0 \text { as } n \rightarrow \infty .
$$


As a consequence of Steps 1 and 2, we can conclude that $N(w): W(w) \rightarrow W(w)$ is a continuous random operator with stochastic domain $W$, and $N(w)(W(w))$ is bounded.

Step 3: For each bounded subset $B$ of $W(w)$ we have

$$
\alpha(N(w) B) \leq \ell \alpha(B) .
$$

Let $w \in \Omega$ be fixed. From Lemmas 2.17 and 2.18, for any $B \subset W$ and any $\epsilon>0$, there exists a sequence $\left\{u_{n}\right\}_{n=1}^{\infty} \subset B$, such that for all $(x, y) \in J$, we have

$$
\begin{aligned}
& \alpha((N(w) B)(x, y)) \\
& =\alpha\left(\left\{\mu(x, y, w)+\int_{0}^{x} \int_{0}^{y} \frac{(x-s)^{r_{1}-1}(y-t)^{r_{2}-1}}{\Gamma\left(r_{1}\right) \Gamma\left(r_{2}\right)} f(s, t, u(s, t, w), w) d t d s ; u \in B\right\}\right) \\
& \leq 2 \alpha\left(\left\{\int_{0}^{x} \int_{0}^{y} \frac{(x-s)^{r_{1}-1}(y-t)^{r_{2}-1}}{\Gamma\left(r_{1}\right) \Gamma\left(r_{2}\right)} f\left(s, t, u_{n}(s, t, w), w\right) d t d s\right\}_{n=1}^{\infty}\right)+\epsilon \\
& \leq 4 \int_{0}^{x} \int_{0}^{y} \alpha\left(\left\{\frac{(x-s)^{r_{1}-1}(y-t)^{r_{2}-1}}{\Gamma\left(r_{1}\right) \Gamma\left(r_{2}\right)} f\left(s, t, u_{n}(s, t, w), w\right)\right\}_{n=1}^{\infty}\right) d t d s+\epsilon \\
& \leq 4 \int_{0}^{x} \int_{0}^{y} \frac{(x-s)^{r_{1}-1}(y-t)^{r_{2}-1}}{\Gamma\left(r_{1}\right) \Gamma\left(r_{2}\right)} \alpha\left(\left\{f\left(s, t, u_{n}(s, t, w), w\right)\right\}_{n=1}^{\infty}\right) d t d s+\epsilon \\
& \leq 4 \int_{0}^{x} \int_{0}^{y} \frac{(x-s)^{r_{1}-1}(y-t)^{r_{2}-1}}{\Gamma\left(r_{1}\right) \Gamma\left(r_{2}\right)} q(s, t, w) \alpha\left(\left\{u_{n}(s, t, w)\right\}_{n=1}^{\infty}\right) d t d s+\epsilon \\
& \leq\left(4 \int_{0}^{x} \int_{0}^{y} \frac{(x-s)^{r_{1}-1}(y-t)^{r_{2}-1}}{\Gamma\left(r_{1}\right) \Gamma\left(r_{2}\right)} q(s, t, w) d s d t\right) \alpha\left(\left\{u_{n}\right\}_{n=1}^{\infty}\right)+\epsilon \\
& \leq\left(4 \int_{0}^{x} \int_{0}^{y} \frac{(x-s)^{r_{1}-1}(y-t)^{r_{2}-1}}{\Gamma\left(r_{1}\right) \Gamma\left(r_{2}\right)} q(s, t, w) d t d s\right) \alpha(B)+\epsilon \\
& \leq \frac{4 q^{*} a^{r_{1}} b^{r_{2}}}{\Gamma\left(1+r_{1}\right) \Gamma\left(1+r_{2}\right)} \alpha(B)+\epsilon \\
& =\ell \alpha(B)+\epsilon .
\end{aligned}
$$

Since $\epsilon>0$ is arbitrary, then

$$
\alpha(N(B)) \leq \ell \alpha(B)
$$

It follows from Lemma 2.19 that for each $w \in \Omega, N$ has at least one fixed point in $W$. Since $\bigcap_{w \in \Omega} \operatorname{int} W(w) \neq \emptyset$ the hypothesis that a measurable selector of int $W$ exists holds. By Lemma 2.19, $N$ has a stochastic fixed point, i.e., the problem (1)-(2) has at least one random solution on $\mathcal{C}$.

Theorem 3.5. Assume that the assumptions $\left(H_{1}\right),\left(H_{2}\right),\left(H_{5}\right)-\left(H_{7}\right)$ hold. Then the random equation (1) is generalized Ulam-Hyers-Rassias stable.

Proof. Let $u: \Omega \rightarrow A C(J)$ be a solution of the inequality (5). By Theorem 3.4, there exists $v$ which is a solution of the random problem (1)-(2). Hence

$$
v(x, y, w)=\mu(x, y, w)
$$




$$
+\int_{0}^{x} \int_{0}^{y} \frac{(x-s)^{r_{1}-1}(y-t)^{r_{2}-1}}{\Gamma\left(r_{1}\right) \Gamma\left(r_{2}\right)} f(s, t, v(s, t, w), w) d t d s ; \quad(x, y) \in J, w \in \Omega .
$$

By differential inequality (5), for each $(x, y) \in J$ and $w \in \Omega$, we have

$$
\begin{aligned}
& \left\|u(x, y, w)-\mu(x, y, w)-\int_{0}^{x} \int_{0}^{y} \frac{(x-s)^{r_{1}-1}(y-t)^{r_{2}-1}}{\Gamma\left(r_{1}\right) \Gamma\left(r_{2}\right)} f(s, t, u(s, t, w), w) d t d s\right\|_{E} \\
& \leq \int_{0}^{x} \int_{0}^{y} \frac{(x-s)^{r_{1}-1}(y-t)^{r_{2}-1}}{\Gamma\left(r_{1}\right) \Gamma\left(r_{2}\right)} \Phi(s, t, w) d t d s .
\end{aligned}
$$

Thus, by $\left(H_{7}\right)$ for each $(x, y) \in J$ and $w \in \Omega$, we obtain

$$
\begin{aligned}
& \left\|u(x, y, w)-\mu(x, y, w)-\int_{0}^{x} \int_{0}^{y} \frac{(x-s)^{r_{1}-1}(y-t)^{r_{2}-1}}{\Gamma\left(r_{1}\right) \Gamma\left(r_{2}\right)} f(s, t, u(s, t, w), w) d t d s\right\|_{E} \\
& \leq \lambda_{\Phi} \Phi(x, y, w) .
\end{aligned}
$$

Hence for each $(x, y) \in J$ and $w \in \Omega$, it follows that

$$
\begin{gathered}
\|u(x, y, w)-v(x, y, w)\|_{E} \\
\leq\left\|u(x, y, w)-\mu(x, y, w)-\int_{0}^{x} \int_{0}^{y} \frac{(x-s)^{r_{1}-1}(y-t)^{r_{2}-1}}{\Gamma\left(r_{1}\right) \Gamma\left(r_{2}\right)} f(s, t, u(s, t, w), w) d t d s\right\|_{E} \\
+\int_{0}^{x} \int_{0}^{y} \frac{(x-s)^{r_{1}-1}(y-t)^{r_{2}-1}}{\Gamma\left(r_{1}\right) \Gamma\left(r_{2}\right)}\|f(s, t, u(s, t, w), w)-f(s, t, v(s, t, w), w)\|_{E} d t d s .
\end{gathered}
$$

From $\left(H_{6}\right)$, for each $(x, y) \in J$ and $w \in \Omega$, we get

$$
\begin{gathered}
\|u(x, y, w)-v(x, y, w)\|_{E} \\
\leq \lambda_{\Phi} \Phi(x, y, w)+\frac{q^{*}}{\Gamma\left(r_{1}\right) \Gamma\left(r_{2}\right)} \int_{0}^{x} \int_{0}^{y}(x-s)^{r_{1}-1}(y-t)^{r_{2}-1}\|u(s, t, w)-v(s, t, w)\|_{E} d t d s .
\end{gathered}
$$

From Lemma 2.20, there exists a constant $\delta=\delta\left(r_{1}, r_{2}\right)$ such that

$$
\begin{aligned}
\|u(x, y, w)-v(x, y, w)\|_{E} & \leq \lambda_{\Phi} \Phi(x, y, w) \\
& +\frac{\delta q^{*} \lambda_{\Phi}}{\Gamma\left(r_{1}\right) \Gamma\left(r_{2}\right)} \int_{0}^{x} \int_{0}^{y}(x-s)^{r_{1}-1}(y-t)^{r_{2}-1} \Phi(s, t, w) d t d s \\
& \leq\left(1+\delta q^{*} \lambda_{\Phi}\right) \lambda_{\Phi} \Phi(x, y, w) \\
& :=c_{f, \Phi} \Phi(x, y, w) .
\end{aligned}
$$

Finally, the random equation (1) is generalized Ulam-Hyers-Rassias stable.

\section{An Example}

Let $E=\mathbb{R}, \Omega=(-\infty, 0)$ be equipped with the usual $\sigma$-algebra consisting of Lebesgue measurable subsets of $(-\infty, 0)$. Given a measurable function $u: \Omega \rightarrow A C([0,1] \times[0,1])$, consider the following partial functional random differential equation of the form

$$
\left({ }^{c} D_{\theta}^{r} u\right)(x, y, w)=\frac{w^{2} e^{-x-y-10}}{1+w^{2}+|u(x, y, w)|} ; \quad \text { a.a. }(x, y) \in J=[0,1] \times[0,1], w \in \Omega,
$$


with the initial conditions

$$
\left\{\begin{array}{l}
u(x, 0, w)=x \sin w ; x \in[0,1], \\
u(0, y, w)=y^{2} \cos w ; y \in[0,1],
\end{array} \quad w \in \Omega\right.
$$

where $\left(r_{1}, r_{2}\right) \in(0,1] \times(0,1]$. Set

$$
f(x, y, u(x, y, w), w)=\frac{w^{2}}{\left(1+w^{2}+|u(x, y, w)|\right) e^{x+y+10}},(x, y) \in[0,1] \times[0,1], w \in \Omega .
$$

The functions $w \mapsto \varphi(x, 0, w)=x \sin w$ and $w \mapsto \psi(0, y, w)=y^{2} \cos w$ are measurable and bounded with

$$
|\varphi(x, 0, w)| \leq 1,|\psi(0, y, w)| \leq 1,
$$

hence, the condition $\left(H_{1}\right)$ is satisfied.

Clearly, the map $(x, y, w) \mapsto f(x, y, u, w)$ is jointly continuous for all $u \in \mathbb{R}$ and hence jointly measurable for all $u \in \mathbb{R}$. Also the map $u \mapsto f(x, y, u, w)$ is continuous for all $(x, y) \in J$ and $w \in \Omega$. So the function $f$ is Carathéodory on $[0,1] \times[0,1] \times \mathbb{R} \times \Omega$.

For each $u \in \mathbb{R},(x, y) \in[0,1] \times[0,1]$ and $w \in \Omega$, we have

$$
|f(x, y, u, w)| \leq 1+\frac{1}{e^{10}}|u| .
$$

Hence the condition $\left(H_{3}\right)$ is satisfied with $p_{1}(x, y, w)=p_{1}^{*}=1$ and $p_{2}(x, y, w)=p_{2}^{*}=$ $\frac{1}{e^{10}}$.

Also, condition $\left(H_{6}\right)$ is satisfied with $q^{*}=\frac{1}{e^{10}}$. Indeed, for each $u, \bar{u} \in \mathbb{R}$ and $(x, y) \in[0,1] \times[0,1]$ and $w \in \Omega$, we get

$$
|f(x, y, u, w)-f(x, y, \bar{u}, w)| \leq \frac{1}{e^{10}}|u-\bar{u}| .
$$

We shall show that condition $\ell<1$ holds with $a=b=1$. Indeed, we have $q^{*}=\frac{1}{e^{10}}$ and for each $\left(r_{1}, r_{2}\right) \in(0,1] \times(0,1]$ we get

$$
\begin{aligned}
\ell & =\frac{4 q^{*} a^{r_{1}} b^{r_{2}}}{\Gamma\left(1+r_{1}\right) \Gamma\left(1+r_{2}\right)} \\
& =\frac{4}{e^{10} \Gamma\left(1+r_{1}\right) \Gamma\left(1+r_{2}\right)} \\
& <1 .
\end{aligned}
$$

Finally, the hypothesis $\left(H_{7}\right)$ is satisfied with

$$
\Phi(x, y, w)=w^{2} x y^{2}
$$

and

$$
\lambda_{\Phi}=\frac{2}{\Gamma\left(2+r_{1}\right) \Gamma\left(3+r_{2}\right)}
$$


Indeed, for each $(x, y) \in[0,1] \times[0,1]$ and $w \in \Omega$, we get

$$
\begin{aligned}
\left(I_{\theta}^{r} \Phi\right)(x, y, w) & =\frac{w^{2} \Gamma(2) \Gamma(3)}{\Gamma\left(2+r_{1}\right) \Gamma\left(3+r_{2}\right)} x^{1+r_{1}} y^{2+r_{2}} \\
& \leq \frac{2 w^{2} x y^{2}}{\Gamma\left(2+r_{1}\right) \Gamma\left(3+r_{2}\right)} \\
& =\lambda_{\Phi} \Phi(x, y, w) .
\end{aligned}
$$

Consequently, Theorem 3.4 implies that the problem (8)-(9) has a random solution defined on $[0,1] \times[0,1]$, and Theorem 3.5 implies that the random equation $(8)$ is generalized Ulam-Hyers-Rassias stable.

\section{References}

[1] S. Abbas, M. Benchohra, Partial hyperbolic differential equations with finite delay involving the Caputo fractional derivative, Commun. Math. Anal. 7 (2009) 62-72.

[2] S. Abbas, M. Benchohra, Fractional order partial hyperbolic differential equations involving Caputo's derivative, Stud. Univ. Babeş-Bolyai Math. 57 (4) (2012) 469479 .

[3] S. Abbas, M. Benchohra, A. Cabada, Partial neutral functional integrodifferential equations of fractional order with delay, Bound. Value Prob. 2012 (2012) $128,15 \mathrm{pp}$.

[4] S. Abbas, M. Benchohra, G.M. N'Guérékata, Topics in Fractional Differential Equations, Springer, New York, 2012.

[5] S. Abbas, M. Benchohra, G.M. N'Guérékata, Advanced Fractional Differential and Integral Equations, Nova Science Publishers, New York, 2015.

[6] S. Abbas, M. Benchohra, J.J. Nieto, Ulam stabilities for impulsive partial fractional differential equations, Acta Univ. Palacki. Olomuc. 53 (1) (2014) 5-17.

[7] S. Abbas, M. Benchohra, S. Sivasundaram, Ulam stability for partial fractional differential inclusions with multiple delay and impulses via Picard operators, J. Nonlinear Stud. 20 (4) (2013) 623-641.

[8] S. Abbas, M. Benchohra, A.N. Vityuk, On fractional order derivatives and Darboux problem for implicit differential equations, Frac. Calc. Appl. Anal. 15 (2012) 168-182.

[9] J. Appell, Implicit functions, nonlinear integral equations, and the measure of noncompactness of the superposition operator, J. Math. Anal. Appl. 83 (1981) 251-263. 
[10] J.M. Ayerbee Toledano, T. Dominguez Benavides, G. Lopez Acedo, Measures of Noncompactness in Metric Fixed Point Theory, Operator Theory, Advances and Applications, vol. 99, Birkhäuser, Basel, Boston, Berlin, 1997.

[11] D. Baleanu, R.P. Agarwal, H. Mohammadi, Sh. Rezapour, Some existence results for a nonlinear fractional differential equation on partially ordered Banach spaces, Bound. Value Prob. 2013 (2013) 112, 10 pp.

[12] D. Bothe, Multivalued perturbation of m-accretive differential inclusions, Isr. J. Math. 108 (1998) 109-138.

[13] M.F. Bota-Boriceanu, A. Petrusel, Ulam-Hyers stability for operatorial equations and inclusions, Analele Univ. I. Cuza Iasi 57 (2011) 65-74.

[14] T.A. Burton, T. Furumochi, A note on stability by Schauder's theorem, Funkcial. Ekvac. 44 (2001) 73-82.

[15] L.P. Castro, A. Ramos, Hyers-Ulam-Rassias stability for a class of Volterra integral equations, Banach J. Math. Anal. 3 (2009) 36-43.

[16] M.A. Darwish, J. Henderson, D. O'Regan, Existence and asymptotic stability of solutions of a perturbed fractional functional-integral equation with linear modification of the argument, Bull. Korean Math. Soc. 48 (3) (2011) 539-553.

[17] M.A. Darwish, J. Henderson, Nondecreasing solutions of a quadratic integral equation of Urysohn-Stieltjes type, Rocky Mountain J. Math. 42 (2) (2012) 545566.

[18] M.A. Darwish, J. Banaś, Existence and characterization of solutions of nonlinear Volterra-Stieltjes integral equations in two vriables, Abstr. Appl. Anal. 2014, Art. ID 618434, 11 pp.

[19] B.C. Dhage, S.V. Badgire, S.K. Ntouyas, Periodic boundary value problems of second order random differential equations, Electron. J. Qual. Theory Diff. Equ. 21 (2009) 1-14.

[20] H.W. Engl, A general stochastic fixed-point theorem for continuous random operators on stochastic domains, J. Math. Anal. Appl. 66 (1978) 220-231.

[21] X. Han, X. Ma, G. Dai, Solutions to fourth-order random differential equations with periodic boundary conditions, Electron. J. Differential Equations 235 (2012) $1-9$.

[22] R. Hilfer, Applications of Fractional Calculus in Physics, World Scientific, Singapore, 2000.

[23] D.H. Hyers, On the stability of the linear functional equation, Proc. Nat. Acad. Sci. 27 (1941) 222-224. 
[24] D.H. Hyers, G. Isac, Th.M. Rassias, Stability of Functional Equations in Several Variables, Birkhäuser, 1998.

[25] S. Itoh, Random fixed point theorems with applications to random differential equations in Banach spaces, J. Math. Anal. Appl. 67 (1979) 261-273.

[26] S.-M. Jung, Hyers-Ulam-Rassias Stability of Functional Equations in Mathematical Analysis, Hadronic Press, Palm Harbor, 2001.

[27] S.-M. Jung, Hyers-Ulam-Rassias Stability of Functional Equations in Nonlinear Analysis, Springer, New York, 2011.

[28] S.-M. Jung, A fixed point approach to the stability of a Volterra integral equation, Fixed Point Theory Appl. 2007 (2007), Article ID 57064, 9 pp.

[29] A.A. Kilbas, H.M. Srivastava, J.J. Trujillo, Theory and Applications of Fractional Differential Equations. North-Holland Mathematics Studies, 204. Elsevier Science B.V., Amsterdam, 2006.

[30] L. Liu, F. Guo, C. Wu, Y. Wu, Existence theorems of global solutions for nonlinear Volterra type integral equations in Banach spaces, J. Math. Anal. Appl. 309 (2005) 638-649.

[31] H. Mönch, Boundary value problems for nonlinear ordinary differential equations of second order in Banach spaces, Nonlinear Anal., Theory Methods Appl. 4 (1980) 985-999.

[32] B.G. Pachpatte, Analytic Inequalities. Recent Advances. Atlantis Studies in Mathematics, 3. Atlantis Press, Paris, 2012.

[33] B.G. Pachpatte, Multidimensional Integral Equations and Inequalities. Atlantis Studies in Mathematics for Engineering and Science, 9. Atlantis Press, Paris, 2011.

[34] T.P. Petru, M.-F. Bota, Ulam-Hyers stabillity of operational inclusions in complete gauge spaces, Fixed Point Theory 13 (2012) 641-650.

[35] T.P. Petru, A. Petrusel, J.-C. Yao, Ulam-Hyers stability for operatorial equations and inclusions via nonself operators, Taiwanese J. Math. 15 (2011) 2169-2193.

[36] I. Podlubny, Fractional Differential Equations, Academic Press, San Diego, 1999.

[37] I. Podlubny, I. Petraš, B.M. Vinagre, P. O'Leary, L. Dorčak, Analogue realizations of fractional-order controllers. Fractional order calculus and its applications, Nonlinear Dynam. 29 (2002) 281-296.

[38] Th.M. Rassias, On the stability of linear mappings in Banach spaces, Proc. Amer. Math. Soc. 72 (1978) 297-300.

[39] I.A. Rus, Ulam stability of ordinary differential equations, Studia Univ. BabesBolyai, Math. LIV (4) (2009) 125-133. 
[40] I.A. Rus, Remarks on Ulam stability of the operatorial equations, Fixed Point Theory 10 (2009) 305-320.

[41] T.T. Soong, Random Differential Equations in Science and Engineering. Academic Press, New York, 1973.

[42] V.E. Tarasov, Fractional Dynamics. Applications of Fractional Calculus to Dynamics of Particles, Fields and Media. Springer, Heidelberg, 2010.

[43] S.M. Ulam, A Collection of Mathematical Problems, Interscience Publishers, New York, 1968.

[44] A.N. Vityuk, A.V. Golushkov, Existence of solutions of systems of partial differential equations of fractional order, Nonlinear Oscil. 7 (3) (2004) 318-325.

[45] A.N. Vityuk, A.V. Mikhailenko, The Darboux problem for an implicit fractionalorder differential equation, J. Math. Sci. 175 (4) (2011) 391-401.

[46] A.N. Vityuk, A.V. Mikhailenko, Darboux problem for differential equation with mixed regularized derivative of fractional order. Nonlinear Stud. 20 no. 4 (2013) 571-580.

[47] J. Wang, L. Lv, Y. Zhou, Ulam stability and data dependence for fractional differential equations with Caputo derivative, Electron. J. Qual. Theory Differ. Equ. 63 (2011) 1-10.

[48] J. Wang, L. Lv, Y. Zhou, New concepts and results in stability of fractional differential equations, Commun. Nonlinear Sci. Numer. Simul. 17 (2012) 25302538 .

[49] W. Wei, X. Li, X. Li, New stability results for fractional integral equation, Comput. Math. Appl. 64 (2012) 3468-3476.

[50] S. Zhang, J. Sun, Existence of mild solutions for the impulsive semilinear nonlocal problem with random effects, Advances in Difference Equations 19 (2014) 1-11.

[51] Y. Zhou, Basic Theory of Fractional Differential Equations, World Scientific, Singapore, 2014.

\section{DOI: $10.7862 /$ rf.2016.1}

\section{Saïd Abbas}

email: abbasmsaid@yahoo.fr

Laboratory of Mathematics,

University of Saïda,

P.O. Box 138, Saïda 20000, Algeria. 
Mouffak Benchohra

email: benchohra@univ-sba.dz

Laboratory of Mathematics,

University of Sidi Bel-Abbes,

P.O. Box 89, Sidi Bel-Abbès 22000, Algeria.

\section{Mohamed Abdalla Darwish}

email: dr.madarwish@gmail.com

Department of Mathematics, Sciences Faculty for Girls, King Abdulaziz University, Jeddah, Saudi Arabia.

Department of Mathematics, Faculty of Science, Damanhour University,

Damanhour, Egypt.

Received 29.05.2016

Accepted 2\%.11.2016 